\title{
Merging of superfluid helium nanodroplets with vortices
}

\author{
José María Escartín $\odot,{ }^{1,2}$ Francesco Ancilotto, ${ }^{3,4}$ Manuel Barranco, ${ }^{5,6}$ and Martí Pi $\oplus^{5,6}$ \\ ${ }^{1}$ Theory of Condensed Matter Group, Cavendish Laboratory, University of Cambridge, 19 JJ Thomson Avenue, \\ Cambridge CB3 OHE, United Kingdom \\ ${ }^{2}$ Catalan Institute of Nanoscience and Nanotechnology (ICN2), CSIC and BIST, Campus UAB, Bellaterra, 08193 Barcelona, Spain \\ ${ }^{3}$ Dipartimento di Fisica e Astronomia "Galileo Galilei" and CNISM, Università di Padova, via Marzolo 8, 35122 Padova, Italy \\ ${ }^{4}$ CNR-IOM, via Bonomea, 265 - 34136 Trieste, Italy \\ ${ }^{5}$ Departament FQA, Facultat de Física, Universitat de Barcelona. Diagonal 645, 08028 Barcelona, Spain \\ ${ }^{6}$ Institute of Nanoscience and Nanotechnology (IN2UB), Universitat de Barcelona, 08028 Barcelona, Spain
}

(Received 21 October 2021; revised 3 January 2022; accepted 4 January 2022; published 13 January 2022)

\begin{abstract}
Within density functional theory, we have investigated the coalescence dynamics of two superfluid helium nanodroplets hosting vortex lines in different relative orientations, which are drawn towards each other by the Van der Waals mutual attraction. We have found a rich phenomenology depending on how the vortex lines are oriented. In particular, when a vortex and antivortex lines are present in the merging droplets, a dark soliton develops at the droplet contact region, which eventually decays into vortex rings. Reconnection events are observed between the vortex lines or rings, leading to the creation of more vortices. Our simulations show the interplay between vortex creation and reconnections, as well as the effect of the droplet surface which pins the vortex ends and, by reflecting short-wavelength excitations produced by the interactions between vortices, strongly affects the droplet final state. Additional vorticity is nucleated in the proximity of surface indentations produced in the course of the dynamics, which in turn interact with other vortices present in the droplets. These effects, obviously absent in the case of bulk liquid helium, show that the droplet surface may act as a multiplier of vortex reconnections. The analysis of the energy spectrum shows that vortex-antivortex ring annihilation, as well as vortex-antivortex reconnections, yields roton bursts of different intensity.
\end{abstract}

DOI: 10.1103/PhysRevB.105.024511

\section{INTRODUCTION}

Droplet merging is a well established subject of investigation in classical fluids as it arises in different fundamental and practical contexts [1-5]. It involves viscous droplets, mostly drawn together by surface tension or Van der Waals mutual attraction. Experiments and models aim at determining, e.g., how the radius of the contact region between drops grows as a function of time after contact, or the final outcome of the interaction between two droplets (merging in one single droplet or separation into two or several), which depends on the size, viscosity, morphology and relative velocity of the colliding droplets, see, e.g., Refs. [6-10] and references therein.

At variance, little is known on the coalescence of drops made of superfluids, for which viscosity is negligible. Experiments on magnetically levitated helium droplets at $0.7 \mathrm{~K}$ temperature have been carried out some time ago by Maris and coworkers [11]. Using a static magnetic field, drops of less than $1 \mathrm{~cm}$ radius were confined and made to collide at velocities as small as a few $\mathrm{cm} / \mathrm{s}$. We have recently simulated this experiment using nanoscopic He droplets within the time-dependent density functional theory (TDDFT) approach [12]. The droplets were initially at rest and drawn together by the mutual Van der Waals (VdW) long-range attraction. It was shown that the radius of the contact region grows as the root square of time after contact, similarly as for low viscosity classical drops [1-3]. This scaling has been also found for the collision of self-bound quantum droplets made of ultradilute Bose mixtures of cold gases [13].

The merging of vortex-free helium droplets has unveiled the appearance of vortex-antivortex ring pairs nucleated at the droplet surface, that either wrap around the coalesced droplet or penetrated into it, eventually annihilating each other yielding an intense roton burst [12]. These effects are most remarkable if one recalls that, initially, no vortices were in the droplets and that droplets were not in relative motion. This calls for studying a more general situation where some vorticity is already present in the coalescent droplets, and constitutes the motivation of the present work. Within TDDFT, we address here the merging of two equal-size superfluid helium droplets at zero temperature, which may host vortex lines with different relative orientations, as schematically represented in Fig. 1.

We shall show that, depending on how droplets are initially prepared, a rich variety of phenomena may occur, as the merging process changes the vorticity of the system in a complex way. One important outcome of our simulations is a dynamical description of vortex interactions and reconnections, which are fundamental ingredients of current studies of quantum turbulence in liquid $\mathrm{He}$ and in cold-gas superfluids, see, e.g., Refs. [14-17] for reviews on the subject. 


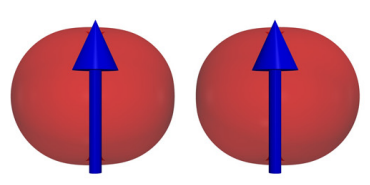

dimer

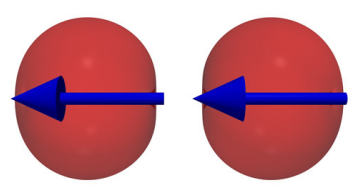

aligned

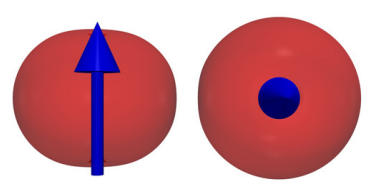

perpendicular

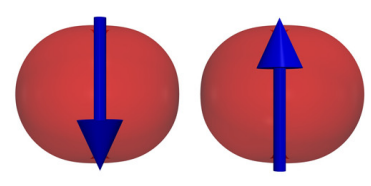

dipole

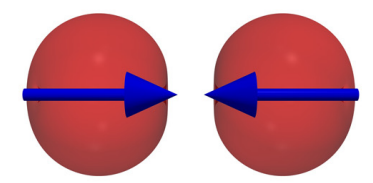

anti-aligned

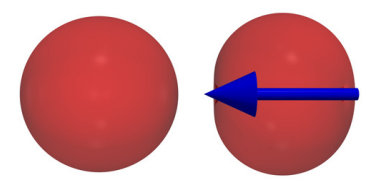

corkscrew

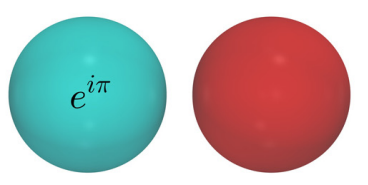

dark soliton

FIG. 1. Schematic view of the merging droplets discussed in this work displaying the different orientation of the vortex lines they host.

Usually, numerical simulations of vortex reconnections in superfluids start from bulklike systems where vortex lines are imprinted which, in order to initiate the vortex-vortex interaction and ensuing reconnection, are subject to additional velocity fields which push them together. Besides, the actual microscopic structure of the vortex cores is most often ignored, and vortex filament models are instead employed. In the present simulations, interactions between vortices are dynamically induced by the spontaneous merging of the droplets; most importantly, the droplet surface provides quite naturally an environment favoring both vortex interaction and proliferation.

This work is organized as follows. In Sec. II, we briefly describe the density functional theory (DFT) approach as applied to liquid ${ }^{4} \mathrm{He}$ and droplets. The results are discussed in Sec. III, complemented with material presented in Ref. [18]; a summary is presented in Sec. IV. Also, multimedia materials accompany this paper showing the real-time dynamics for the merging processes addressed in Ref. [18] This material constitutes an important part of this work, since often it is only by viewing how a complex process unfolds in time that one captures important physical details which would otherwise escape in a written account.

\section{METHOD}

The DFT approach, in its static and time-dependent formulations, has been probed to be a realistic method for studying a strongly correlated quantum fluid as liquid helium [19]; it has been shown to sustain complex dynamical mechanisms, such as vortex nucleation and vortex-density waves interactions and, as shown in the following, vortex reconnections. The Gross-Pitaevskii (GP) approach [20] has been also shown to sustain these effects in dilute cold gases. In particular, slow collisions of two confined Bose-Einstein condensates (BEC) in both ground and exited states, have been addressed within this approach [21].

Previous TDDFT simulations have shown that impurities impinging nanoscopic superfluid He droplets nucleate vortex rings and vortex loops that start and end on the droplet surface $[22,23]$. Other TDDFT simulations have addressed the capture of impurities by vortex-hosting droplets [24], much as it happens in the experiments where captured impurities form clusters along the vortex cores, helping detect the presence of vortices $[25,26]$.

We provide here a basic account of the DFT approach to help discuss the results presented in this paper, and refer the interested reader to Ref. [19] for a detailed description of the method.

\section{A. Statics}

Within DFT, the total energy of a ${ }^{4} \mathrm{He}_{N}$ droplet at zero temperature is written as a functional of a complex effective wave function $\Psi(\mathbf{r})$ related to its atomic density by $\rho(\mathbf{r})=|\Psi(\mathbf{r})|^{2}$. As a first step in the study of droplet merging, the equilibrium configuration of an isolated helium droplet is obtained by solving the static DFT equation for $\Psi(\mathbf{r})$

$$
\left\{-\frac{\hbar^{2}}{2 m} \nabla^{2}+\frac{\delta \mathcal{E}_{c}}{\delta \rho}\right\} \Psi(\mathbf{r}) \equiv \mathcal{H}[\rho] \Psi(\mathbf{r})=\mu \Psi(\mathbf{r}),
$$

where $\mu$ is the ${ }^{4} \mathrm{He}$ chemical potential and $\mathcal{E}_{c}[\rho]$ is the correlation energy. In this study, the number of helium atoms in the droplet is $N=500$, and the results we present have been obtained using the 4He-DFT BCN-TLS computing package [27]. Details on how Eq. (1) is solved can be found in Refs. [19,28] and references therein. Briefly, we work in cartesian coordinates, with the effective wave function $\Psi(\mathbf{r})$ defined at the nodes of a three-dimensional (3D) grid inside a calculation box large enough to accommodate the droplet in such a way that the He density is sensibly zero on the box surface. Periodic boundary conditions are imposed which allow to use the fast Fourier transform (FFT) to carry out the convolutions needed to obtain the DFT mean field $\mathcal{H}[\rho]$. The differential operators in $\mathcal{H}[\rho]$ are approximated by 13-point formulas and the space step is $0.4 \AA$.

The correlation energy functional $\mathcal{E}_{c}[\rho]$ has been taken from Ref. [29]. This functional is finite-range and includes nonlocal effects which are needed to describe quantitatively the response of the superfluid at the scale of the vortex core radius [30], providing an accurate description of the vortex core structure and of the density modulations around it [31]. These density modulations have been interpreted as a cloud of bound, virtual rotons, embodied in the phase of the vortex wave function [32]. It has been furthermore suggested that these virtual rotons may be converted into real ones following vortex-antivortex annihilations, thus making vortex tangles a potential source of nonthermal rotons [32]. 
A vortex line along a diameter of the ${ }^{4} \mathrm{He}_{500}$ droplet can be easily generated by solving Eq. (1) with a proper form for the starting wave function. For instance, the following choice

$$
\Psi_{\text {start }}(\mathbf{r})=\rho_{0}^{1 / 2}(\mathbf{r})\left[\frac{x+t y}{\sqrt{x^{2}+y^{2}}}\right],
$$

where $\rho_{0}(\mathbf{r})$ is the vortex-free droplet density. During the iterative solution of Eq. (1) the vortex core structure changes to provide at convergence the lowest energy configuration, i.e. a vortex line along the $z$ axis with positive (counter clockwise) $n=1$ circulation number and a vortex core size (i.e., the radius of the region where the density drops to zero) of the order of $1 \AA$, see, e.g., Refs. [30,33]. A vortex line with $n=-1$ circulation number (antivortex) is instead generated by taking in the numerator of Eq. (2) the combination $x-\imath y$; vortices along the $x$ and $y$ axes can be generated by expressions similar to Eq. (2).

These vortex states are excited droplet states, eigenstates of the angular momentum about the chosen diameter, with eigenvalue $N \hbar$, i.e., $500 \hbar$ in the present case. The total energy of the ${ }^{4} \mathrm{He}_{500}$ droplet is $-2474.6 \mathrm{~K}$, and that of the ${ }^{4} \mathrm{He}_{500}$ droplet hosting a straight vortex line along a diameter is $-2381.8 \mathrm{~K}$, corresponding to an excitation energy of $92.7 \mathrm{~K}$.

\section{B. Real time dynamics}

Once the structure of the isolated droplet has been obtained, it is used to prepare the initial state for the merging. In all cases, we place two droplets on the $x$ axis such that their center-of-mass are at a $d=41 \AA$ distance. We recall that the droplet sharp density surface, defined by a sphere of radius $R=r_{0} N^{1 / 3}$ with $r_{0}=2.22 \AA$ [34], is $R=17.6 \AA$ for a $N=500$ droplet, so that the sharp density surfaces of the vortex-free droplets are about $6 \AA$ apart.

In classical droplet-merging calculations, the initial condition consists of two droplets whose sharp-surfaces are touching, connected by tiny bridge joining them [1]. In our case, the bridge appears naturally, caused by the VdW mutual attraction. This attraction is absent in any GP approach based on an atom-atom contact interaction, hence some initial velocity has to be provided to the droplets or, alternatively, the confining potential has to be modified in order to trigger the process [13,21].

In our case, the combination

$$
\Psi(\mathbf{r}, t=0)=\Psi_{L}\left(\mathbf{r}-\frac{d}{2} \hat{\mathbf{x}}\right)+\Psi_{R}\left(\mathbf{r}+\frac{d}{2} \hat{\mathbf{x}}\right)
$$

is taken as initial configuration for the dynamics, and the left $\left(\Psi_{L}\right)$ and right $\left(\Psi_{R}\right)$ components may host vortices with given orientations. The TDDFT dynamics is governed by the equation

$$
i \hbar \frac{\partial}{\partial t} \Psi(\mathbf{r}, t)=\left[-\frac{\hbar^{2}}{2 m} \nabla^{2}+\frac{\delta \mathcal{E}_{c}}{\delta \rho}\right] \Psi(\mathbf{r}, t),
$$

which is solved as outlined in Refs. [19,28] using the computing package of Ref. [27]. Most simulations span 600 ps of total elapsed time, with a time step of $1 \mathrm{fs}$.

We recall that if the effective wave function is written as

$$
\Psi(\mathbf{r}, t)=\Phi(\mathbf{r}, t) \exp [\imath \mathcal{S}(\mathbf{r}, t)]
$$
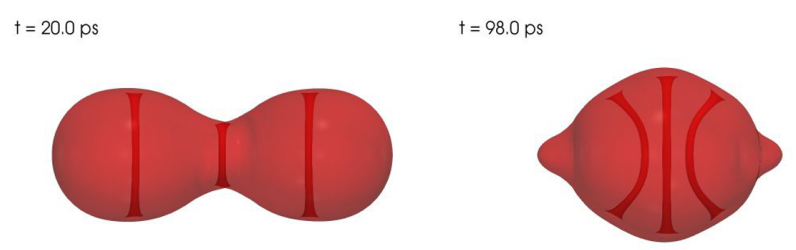

$t=120.0 \mathrm{ps}$

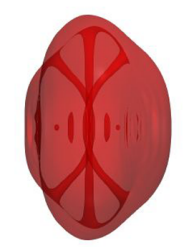

$\dagger=160.0 \mathrm{ps}$

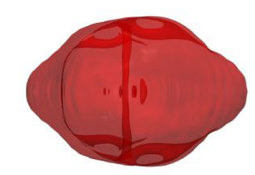

$\dagger=229.0 \mathrm{ps}$

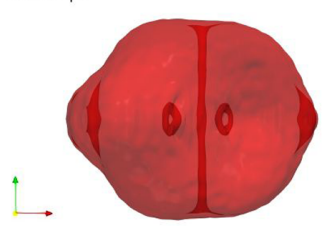

$\dagger=308.0 \mathrm{ps}$

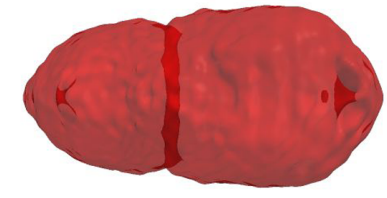

FIG. 2. Merging of two ${ }^{4} \mathrm{He}_{500}$ droplets, each initially hosting a vortex line perpendicular to the merging direction with equal circulation number (vortex dimer, $\langle L\rangle=1000 \hbar$ ). The 3D frames are labeled by the time in picoseconds.

with $\Phi$ and $\mathcal{S}$ real functions, then the particle current density is

$$
\begin{aligned}
\mathbf{j}(\mathbf{r}, t)= & -\frac{l \hbar}{2 m}\left[\Psi^{*}(\mathbf{r}, t) \nabla \Psi(\mathbf{r}, t)-\Psi(\mathbf{r}, t) \nabla \Psi^{*}(\mathbf{r}, t)\right] \\
& =\rho(\mathbf{r}, t) \mathbf{v}(\mathbf{r}, t)
\end{aligned}
$$

with $\rho(\mathbf{r}, t)=\Phi^{2}(\mathbf{r}, t)$ and $\mathbf{v}(\mathbf{r}, t)=\hbar \nabla \mathcal{S}(\mathbf{r}, t) / m$; the phase $\mathcal{S}(\mathbf{r}, t)$ thus represents the velocity potential field except at the vortex line, where the ${ }^{4} \mathrm{He}$ density is zero.

\section{RESULTS}

\section{A. Merging dynamics}

In the vortex-free case, coalescence smoothly proceeds by the development of a tiny, low density bridge connecting the two droplets in about 4-5 ps, which eventually becomes a high density neck [12]. The time evolution of the contact region is dramatically affected by the presence of vortices in the droplets and the interplay between their associated flows [18].

Figure 2 shows snapshots of the merging droplets in the case of two parallel vortices, one in each droplet, perpendicular to the merging direction and with the same quantum circulation (vortex dimer, see Fig. 1), which has a total angular momentum $\langle L\rangle=1000 \hbar$. The 3D droplets are represented by drawing their sharp density surfaces, defined as the loci where the helium density equals that of bulk liquid at zero temperature and pressure divided by two, namely $0.0109 \AA^{-3}$.

At $t=20 \mathrm{ps}$, a vortex line parallel to the original ones appears in the neck region connecting the merging droplets, see Fig. 2. Since angular momentum is strictly conserved, its initial value is shared between the original vortices, the nucleated one and the surface capillary waves which appear in the merged droplet. Intuitively, the vortex nucleated at the 

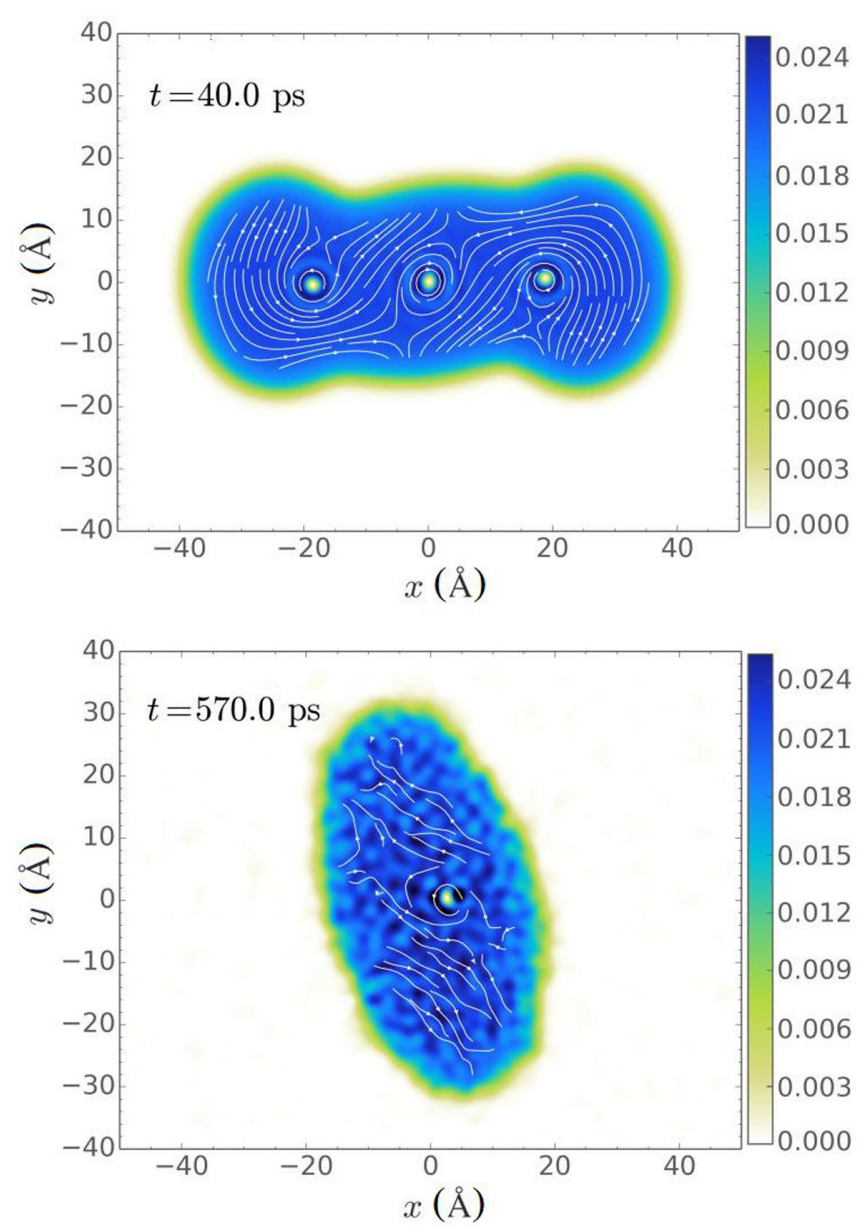

FIG. 3. Snapshots of the 2D density in the $x-y$ plane for the merging of two ${ }^{4} \mathrm{He}_{500}$ droplets, each initially hosting a vortex line along the $z$ axis with the same circulation number (vortex dimer). (Top) $t=40 \mathrm{ps}$, displaying the cross section of the two initial vortex, and that of the nucleated antivortex between them. (Bottom) Same as top at $t=570 \mathrm{ps}$. Superposed to the density are circulation lines of the superfluid flow. The color bar indicates the value of the helium atom density in $\AA^{-3}$.

neck should have a circulation opposite to the original ones [21]. We show in Fig. 3 the circulation lines of the superfluid flow at $t=40 \mathrm{ps}$ in the $x-y$ plane.

At $t=98 \mathrm{ps}$, the original vortex lines have experienced a large bending in order to hit perpendicularly the droplet surface, see Fig. 2. As in the vortex-free case, two protrusions appear on the droplet surface, whose subsequent collapse in the body of the droplet launches a series of vortex rings. The appearance of surface protrusions is frequent in classical droplets collisions [7-10]. What makes protrusions unique in the case of ${ }^{4} \mathrm{He}$ droplets is that they act as nucleation sites of quantized vortex rings. Surface protrusions have also been identified in head-on collisions of self-bound quantum droplets $[13,35]$, but no vortex ring nucleation has been reported in these studies. For these droplets made of ultradilute Bose-gase mixtures, a minimum number of atoms is needed to develop any binding, and the surface tension is very small. As a result, once created the protrusions evaporate almost immediately instead of being re-adsorbed within the merg- ing droplet, hindering the nucleation of vortex rings. The helium-drop mechanism should also work for colliding classical droplets, but we are not aware that nonquantized vortex ring nucleations have been reported in droplets collisions.

The frame in Fig. 2 corresponding to $t=120$ ps shows the appearance of a vortex tangle and of vortex rings as well. The disentanglement of the vortex lines following their mutual crossing yields vortex loops, as shown, e.g., at $t=160$ ps. The dynamic evolution also yields vortex reconnections and the nucleation of vortex rings, as displayed at $t=229$ ps. These vortex rings eventually collide with the droplet surface and evanesce [18]. Finally, a single vortex line emerges, as displayed at $t=308 \mathrm{ps}$, which remains till the end of the simulation while the droplet is still in a highly excited state. The two-dimensional (2D) density on the $x-y$ plane corresponding to the configuration at $t=570 \mathrm{ps}$ is shown in the bottom panel of Fig. 3.

It is worth noticing the presence of helical Kelvin modes along the vortex core, that can only appear if it has some transverse extension. Helical Kelvin modes have also been found in the capture of impurities by vortex lines in helium droplets [24]. Superposed to this complex vortex dynamics arising from surface deformations, the merged droplet undergoes large amplitude oscillations [12].

Figure 3 shows that, besides circulation lines that wrap around the vortex cores, there are others that hit the droplet surface and correspond to surface capillary waves. Surface capillary waves are ubiquitous in deformed superfluid droplets and carry a part-sometimes sizable-of the angular momentum of the droplet [26,36,37].

We have next addressed the coalescence of droplets in the case of two parallel vortices, one in each droplet, perpendicular to the merging direction and with opposite quantum circulation (vortex dipole, see Fig. 1), which has a total angular momentum $\langle L\rangle=0$. Since the phase difference between the superfluid droplets at the touching point is $\pi$, a dark soliton is expected to appear at the contact region. We recall that a dark soliton is a structure (a disk in the present case) made of nodal points of the effective wave function $\Psi(\mathbf{r}, t)$ were the real and imaginary parts are both zero [20]; this is at variance with vortices, where the nodal points form a line.

Bright solitons in thin ${ }^{4} \mathrm{He}$ films adsorbed on solid substrates have been observed [38-41], and also in bulk superfluid ${ }^{4} \mathrm{He}[42,43]$; so far, dark solitons have not been detected in superfluid ${ }^{4} \mathrm{He}$. Instead, they have been observed in BECs [44-48]. While a soliton in 1D is stable, the presence of a transverse dimension causes its decay through the snake instability mechanism [49,50]. In Ref. [51], the time evolution and decay of a dark soliton was observed. The cascade of solitonic excitations arising from the snaking of a planar soliton in BEC has recently been observed [52].

Figure 4 shows snapshots of the merging of the dipole configuration and the appearance of a dark soliton, fully developed at $t=85 \mathrm{ps}$. The droplets are prevented from merging for about $130 \mathrm{ps}$ because the phase difference makes the touching plane a nodal surface. The dark soliton in Fig. 4 is found to undergo a snake instability, decaying into vortex rings that in turn decay into a vortex-antivortex tangle which can be observed in the $t=130 \mathrm{ps}$ frame [18]. Upon vortex reconnections, a distorted vortex ring appears at $t=165$ ps. 

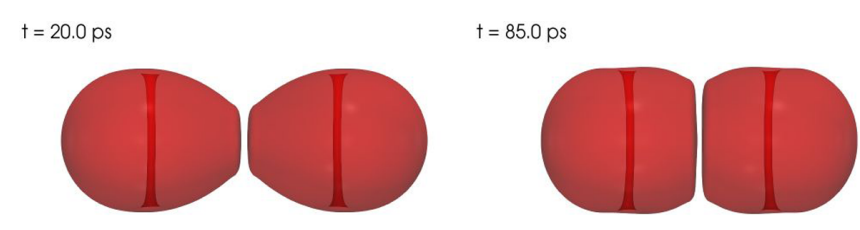

$t=130.0 \mathrm{ps}$

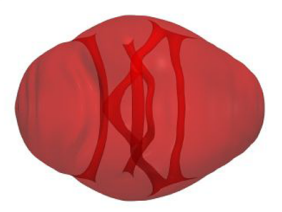

$\dagger=165.0 \mathrm{ps}$

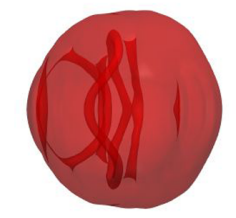

$\dagger=241.0 \mathrm{ps}$
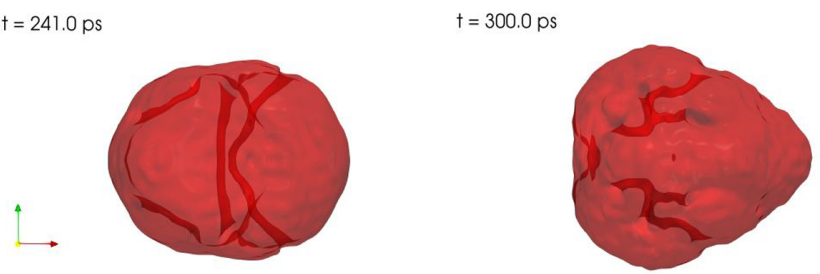

FIG. 4. Merging of two ${ }^{4} \mathrm{He}_{500}$ droplets, each initially hosting a vortex line along the $z$ axis with opposite circulation number (vortex dipole, $\langle L\rangle=0$ ). The $3 \mathrm{D}$ frames are labeled by the time in picoseconds.

Until the end of the simulation, vorticity appears in the bulk of the excited merged droplet, which is very deformed and has many bumpy textures on its surface, as seen, e.g., in the frame at $t=300 \mathrm{ps}$. It can be seen that in the dipole case the merging yields many vortex reconnections [18]; at variance, in the dimer case the vortex that appears as soon as the droplets touch remains stable for a rather long period of time.

A dark soliton similar to the one described before can be created by starting the real time evolution from the effective wave function $\Psi_{0}(\mathbf{r}-d / 2 \hat{\mathbf{x}})+e^{l \pi} \Psi_{0}(\mathbf{r}+d / 2 \hat{\mathbf{x}}), \Psi_{0}$ being the vortex-free effective wave function (see Fig. 1) [18]. A similar preparation for two colliding confined BECs was made in Ref. [21]. As in the vortex dipole case just described, we have found that the dark soliton decays by vortex rings nucleation, which in turn decay into a vortex tangle.

When vortices are oriented perpendicularly to each other and to the merging direction as well (see Fig. 1), droplet coalescence yields a vortex line at the contact region forming an angle of $\pi / 4$ with respect to the original ones. The nucleated vortex is less robust than that appearing in the dimer case, which is aligned with the original ones for quite some time [18]. For this reason, although the time evolution of the merging process is rather similar for both geometries, we have found that vortices become more entangled and yield more reconnections and splittings in the perpendicular than in the dimer case.

We have also addressed the merging of droplets with vortices oriented in other directions [18]. In particular, configurations where two vortices are aligned with the merging direction, either with the same circulation (aligned vortices, see Fig. 1), or with opposed circulation (antialigned vortices, see Fig. 1).
In the aligned case, the simulation has unveiled an unusual scenario. Whereas the final state is expectable--a single vortex line arising from the connection of the two aligned vortices-a series of vortex-antivortex ring pairs are dynamically nucleated at the surface protrusions as in the vortex-free merging [12]. These rings are pierced by the vortex line, which prevents them from shrinking, and guides them till they collide and annihilate at the center of the merged droplet producing a roton burst.

The antialigned case has also yielded its own peculiarities [18]. The droplet coalescence produces two new vortex lines perpendicular to the merging direction, forming a cross pattée (similar to the Maltese cross) with opposite circulations such that the total angular momentum in the merged droplet is kept equal to zero. The collapse of the surface protrusions yields the usual vortex-antivortex ring pairs, pierced again by the antialigned vortex lines. The annihilation of the vortex rings produces helical Kelvin modes along the vortex lines which have been nucleated perpendicularly to the merging direction, clearly visible before the cross structure breaks down into a vortex-antivortex pair whose constituents reconnect, forming a large vortex ring nearly occupying the cross section of the merged droplet before breaking down again into a vortexantivortex pair, and so on. These splittings and reconnections produce roton bursts of different intensities [18].

Finally, we have studied the coalescence of a vortex-free droplet with a droplet hosting a vortex line aligned with the merging direction. This simulation has been motivated by a recent work [53], where two laterally confined cylindrical cold-gas BECs are kept apart by a planar potential barrier between them. One of the BECs is vortex-free and the other hosts a vortex line along the symmetry axis. Suddenly, they are put in contact by releasing the barrier, merging in the direction of the vortex line. A striking result found in this work, is that a "corkscrew" structure emerges at the BECs interface by which angular momentum is transferred from the vortex-hosting to the vortex-free BEC: the vortex line deforms adopting the shape of a corkscrew, penetrating into the vortex-free BEC.

In the merging of superfluid He droplets, the contact region is not planar and droplets deform laterally, so it is interesting to see how the scenario changes. For both systems, the evolution of the vortex line during the first stages of the simulation is similar [18]; since a vortex line cannot end in the bulk of the superfluid, it bends off the merging axis in order to hit perpendicularly the droplet surface. The subsequent evolution is however very different, as the surface of the ${ }^{4} \mathrm{He}$ droplet is dramatically deformed by the merging process and the behavior of the vortex line is erratic, whereas in the confined BECs its time evolution is guided by the lateral confinement, to which the vortex line has to hit perpendicularly. Eventually, both dynamics become similar again, with the original vortex line cut into several vortex loops and the appearance of a very distorted superfluid density.

\section{B. Vorticity and energy spectrum}

Detecting vorticity, defined as $\omega(\mathbf{r}, t)=\nabla \times \mathbf{v}(\mathbf{r}, t)$ [54], is not a trivial task, since it is singular on the vortex lines and zero outside them (a Dirac delta function) [20]. A more 


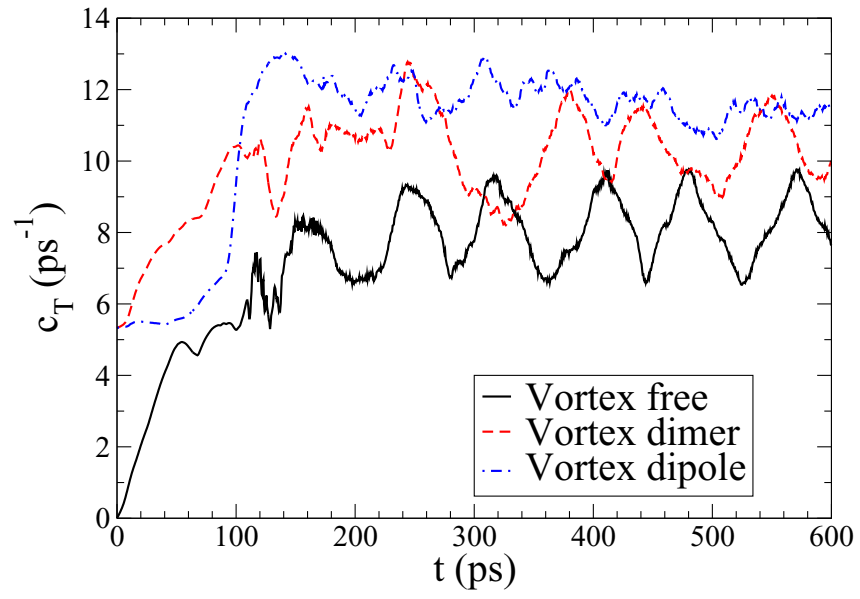

FIG. 5. Space integral of $\left\|\omega_{\mathrm{ps}}(\mathbf{r}, t)\right\|\left(c_{T}(t)\right.$ function) as a function of time for the merging of two ${ }^{4} \mathrm{He}_{500}$ droplets.

convenient way to identify regions of potentially nonzero vorticity in the superfluid is to calculate the curl of the atom current density, $\omega_{\mathrm{ps}}(\mathbf{r}, t)=\nabla \times \mathbf{j}(\mathbf{r}, t)$, called pseudovorticity [55]. Plots of the isosurfaces $\left\|\omega_{\mathrm{ps}}(\mathbf{r}, t)\right\|=C$ allow to visualize regions of nonzero pseudovorticity [12], that may be subsequently analyzed seeking for vorticity inside them.

Similarly, the integrated quantity

$$
c_{T}(t)=\int d \mathbf{r}\left\|\omega_{\mathrm{ps}}(\mathbf{r}, t)\right\|
$$

helps identify the appearance of pseudovorticity in time. Figure 5 shows the function $c_{T}(t)$ for the vortex dimer and vortex dipole droplets merging. It starts at a common nonzero value, as $c_{T}(t)$ does not depend on the relative orientation of the vortices. For the sake of comparison, the result corresponding to the vortex-free droplet merging is also shown. In this case, the steadily raising of $c_{T}(t)$ up to $t \sim 50 \mathrm{ps}$ is due to the nucleation of vortex-antivortex ring pairs at the contact region, that slip on the surface of the coalescent droplets [12]. It was show that quantized ring vortices appeared on the surface of the merged droplet up to much later times, of the order of 115 ps.

In the dimer case, the observed steadily increase of $c_{T}(t)$ up to $t \sim 100 \mathrm{ps}$ is mainly due to the nucleation of the antivortex line in the merging region, with some contribution from the vortex-antivortex ring pairs as in the vortex-free case. In both cases, $c_{T}(t)$ has contributions from the vortex rings nucleated at the surface protrusions.

In the dipole case, Fig. 5 shows that vorticity is nearly constant up to about $t=50 \mathrm{ps}$, slowly increasing during the next $40 \mathrm{ps}$ due to vortex-antivortex ring pairs nucleated in the low density region close to the nodal plane. The sharp rise at about $t=90 \mathrm{ps}$ is produced by the decay of the dark soliton into quantized vortex rings, whose decay yields vortex-antivortex pairs. From this time on, pseudovorticity remains fairly constant during the elapsed time of the simulation (600 ps).

Figure 5 and a similar one in Ref. [18] for the aligned and antialigned vortex orientations, displays conspicuous vorticity oscillations. From the analysis of the real-time evolution of the merging process [18], it appears that the configura-

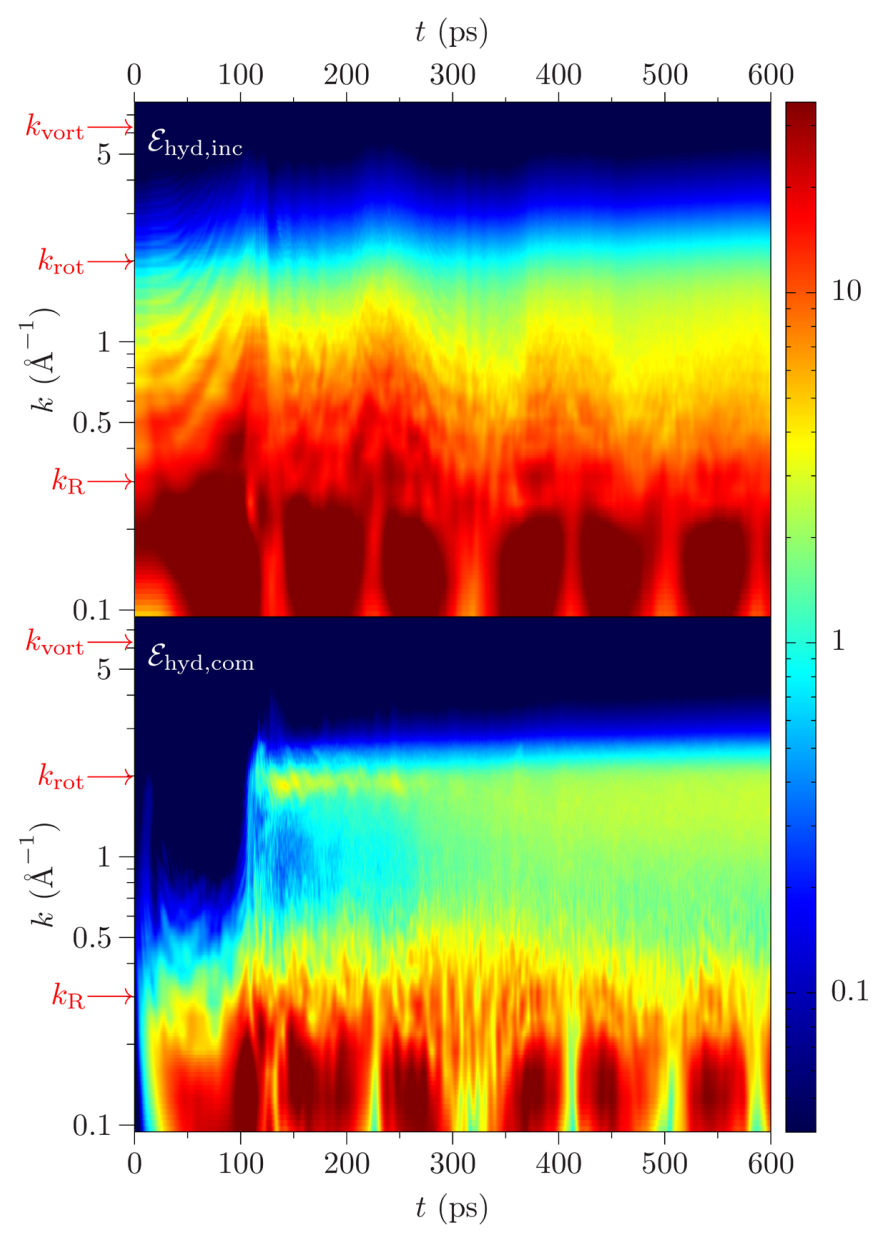

FIG. 6. Energy spectrum $\mathcal{E}_{\text {hyd }}(k, t)(\mathrm{K} \AA)$ for the vortex dimer. (Top) Incompressible part and (bottom) compressible part.

tions associated with the maxima in $c_{T}(t)$ correspond to the more compact, more axisymmetric-like configurations of the merged droplet, while the minima correspond to the more elongated, less axisymmetric-like configurations. Therefore we attribute tentatively the oscillations in $c_{T}(t)$ to the interplay between vortex lines and capillary waves: while they share the angular momentum deposited in the merged droplet, only vortex lines carry vorticity. Since capillary waves only appear in nonaxisymmetric configurations, vortices should be more abundant in compact configurations to conserve the angular momentum, hence increasing the $c_{T}(t)$ value. This reasoning cannot be applied to the other cases.

It is worth noticing that the surface of the merged droplet evolves from smooth to rough. This roughness is remarkably similar to that found on the surface of classical fluids-ethanol and water-in low gravity [56], and it is a hallmark of capillary (classical) wave turbulence [57]. Indeed, nucleation of vortex lines and rings and their reconnections, as well as their subsequent annihilation during the merging, are expected to be a source of quantum turbulence. Vortex ring emission and the possible transition to chaotic turbulent regime occurring when impurities move inside a superfluid has been previously studied within the time-dependent GP approach in both superfluid ${ }^{4} \mathrm{He}$ and BECs [58-65]. Although the GP equation is too simple a model for superfluid helium, it has 


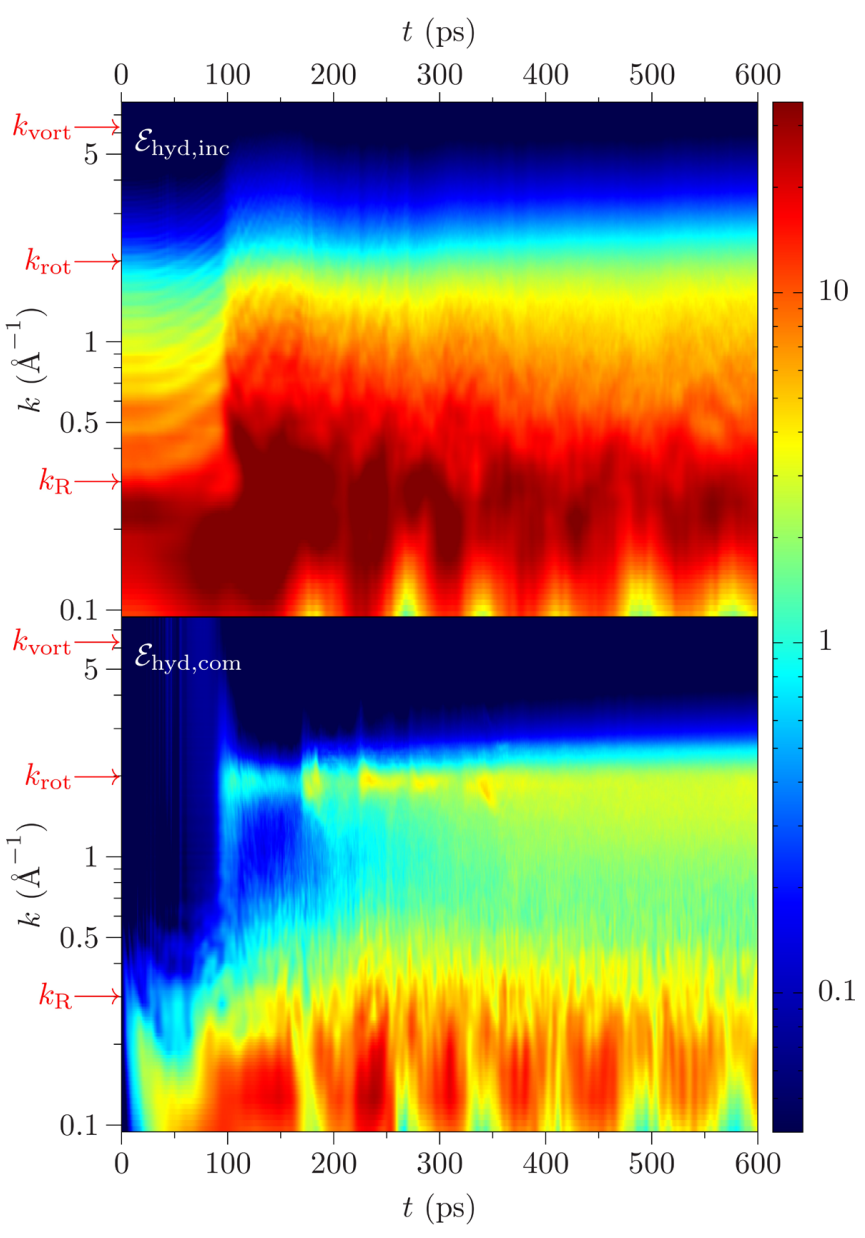

FIG. 7. Energy spectrum $\mathcal{E}_{\text {hyd }}(k, t)(\mathrm{K} \AA)$ for the vortex dipole. (Top) Incompressible part and (bottom) compressible part.

been shown to reproduce the dynamics of vortex ring emission for an electron moving in the liquid at zero temperature, as well as the inherent symmetry breaking of the solution due to the emerging instability, see, e.g., Ref. [59] and references therein.

Turbulence is usually addressed with the aid of the kinetic energy spectrum whose dependence upon the wave number $k$ allows to distinguish different regimes that are relevant for characterizing it [15]. The kinetic energy of the superfluid is written as

$$
E_{\mathrm{kin}}(t)=\frac{\hbar^{2}}{2 m} \int d \mathbf{r}|\nabla \sqrt{\rho(\mathbf{r}, t)}|^{2}+\frac{m}{2} \int d \mathbf{r} \frac{\mathbf{j}^{2}(\mathbf{r}, t)}{\rho(\mathbf{r}, t)},
$$

where the first term is the quantum pressure while the second is the usual hydrodynamic kinetic energy $E_{\text {hyd }}$. In Fourier space, $E_{\text {hyd }}$ is rewritten as

$$
E_{\text {hyd }}(t)=4 \pi \int_{0}^{\infty} d k \mathcal{E}_{\text {hyd }}(k, t),
$$

where the energy spectrum $\mathcal{E}_{\text {hyd }}(k, t)$ is the spherical average in $\mathbf{k}$ space $[58,66,67]$

$$
\mathcal{E}_{\text {hyd }}(k, t)=\frac{m}{2}(2 \pi)^{3} \frac{k^{2}}{4 \pi} \int_{\Omega_{k}} d \Omega_{k}\left|\frac{\widetilde{\mathbf{j}}}{\sqrt{\rho}}(\mathbf{k}, t)\right|^{2} .
$$

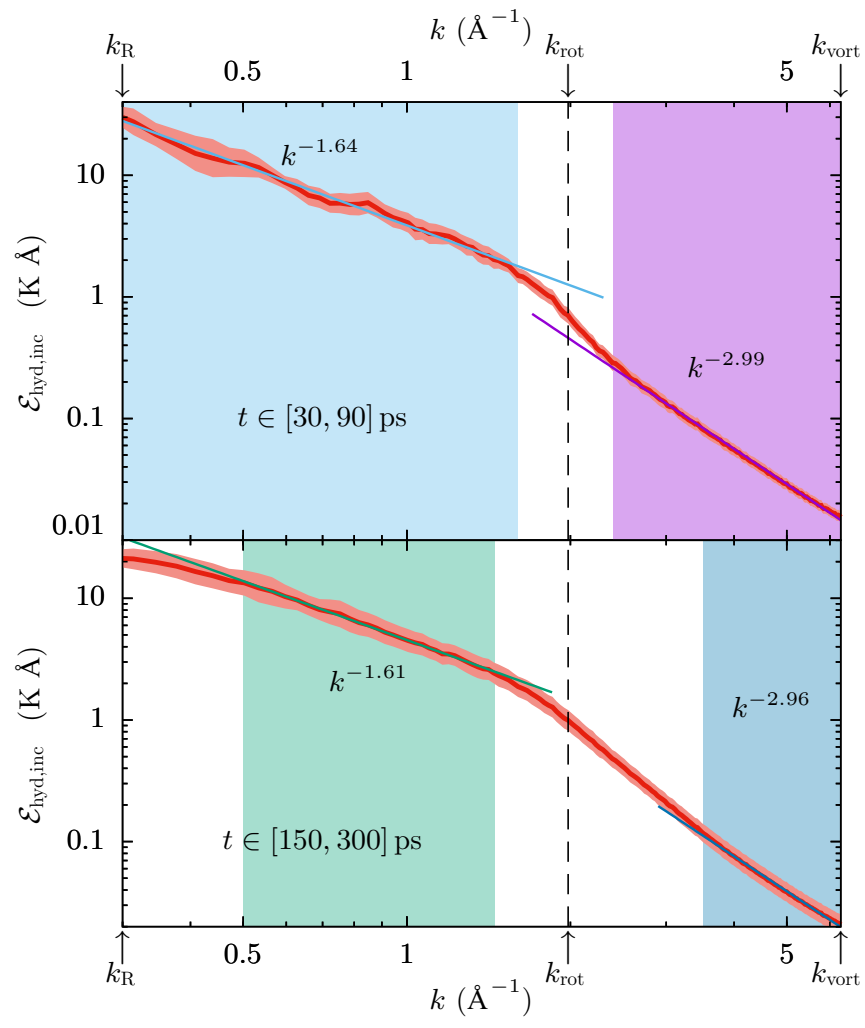

FIG. 8. Power-law analysis of time averages of the incompressible energy spectrum for the dimer configuration. Each of the two panels display, in $\log -\log$ scales, the time averaged spectrum $\mathcal{E}(k)$ (red line) and the range within a standard deviation (light red) for every $k$, as well as the power laws determined by weighted fits over the shadowed $k$ intervals and indicated time intervals. (Top) $k^{-1.64}$ and $k^{-2.99}$ for $t \in[30,90]$ ps. (Bottom) $k^{-1.61}$ and $k^{-2.96}$ for $t \in[150,300]$ ps.

$\mathcal{E}_{\text {hyd }}(k, t)$ is decomposed it into a divergence-free (incompressible) part, related to vorticity, and a compressible part [66], related to density waves, which are analyzed separately [68]. $k$ values relevant for the discussion are $k_{\mathrm{vort}}=2 \pi / a=$ $6.3 \AA^{-1}, a$ being the vortex core radius $\sim 1 \AA ; k_{\mathrm{R}}=2 \pi / R=$ $0.3 \AA^{-1}$, where $R$ is the radial dimension of the droplet, and the roton wave vector $k_{\text {rot }}=1.98 \AA^{-1}$. The wave numbers $k_{\mathrm{R}}, k_{\mathrm{rot}}$, and $k_{\mathrm{vort}}$ are within the range of $k$ values spanned by the FFT given the space step and size of the calculation box. Figure 6 shows both components of $\mathcal{E}_{\text {hyd }}(k, t)$ for the vortex dimer, which periodically display bright regions in the small $k$ region $\left(k \lesssim k_{\mathrm{R}}\right)$ in phase with the oscillations of the shape of the merged droplet. Figure 7 shows the results for the vortex dipole.

In the compressible component (bottom panel in both figures), a series of bright spots appears arising from rotons emission [12]. The simultaneous analysis of the droplet densities [18] and the energy spectrum shows that roton bursts originate from three mechanisms, namely, the annihilation of vortex-antivortex rings, which yields the more intense peaks, vortex-antivortex reconnections, and vortex ring annihilation when they hit the droplet surface. The peaks spread with time, being clearly visible during several hundred picoseconds more. It is worth noticing that in a recent paper, the head-on 


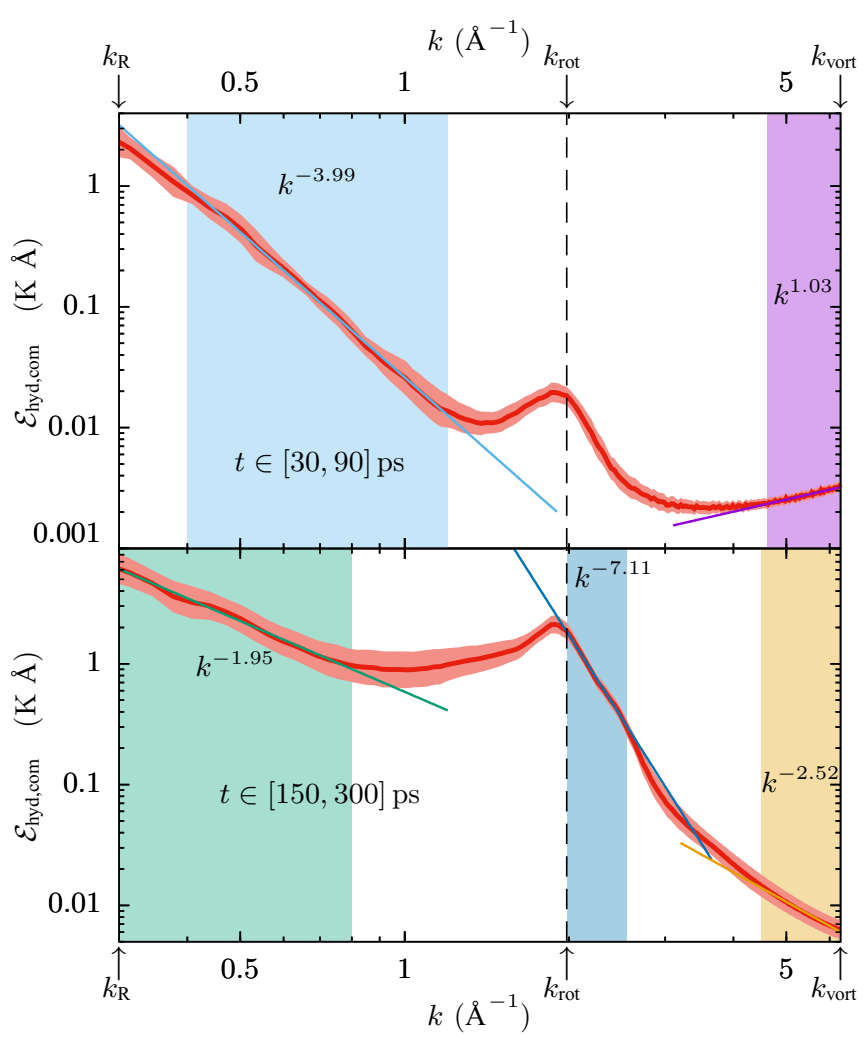

FIG. 9. Power-law analysis of time averages of the compressible energy spectrum for the dimer configuration. Each of the two panels display, in log-log scales, the time averaged spectrum $\mathcal{E}(k)$ (red line) and the range within a standard deviation (light red) for every $k$, as well as the power laws determined by weighted fits over the shadowed $k$ intervals and indicated time intervals. (Top) $k^{-3.99}$ and $k^{1.03}$ for $t \in[30,90]$ ps. (Bottom) $k^{-1.95}, k^{-7.11}$, and $k^{-2.52}$ for $t \in[150,300]$ ps.

collision of a pair of smoke vortex-antivortex rings has been visualized [69], as well as their ultimate breakdown yielding a burst of sound waves similar to that we have found for rotons, stressing its connection to the appearance of classical turbulence.

As in Ref. [12], we have carried out power-law $\left(k^{\alpha}\right)$ analyses for selected time average intervals of the compressible and incompressible spectra for the droplet mergings addressed in this work. Figures 8 and 9 show the power-law analysis for the dimer configuration. The first time interval (top panels in the figures, 30 to $90 \mathrm{ps}$ ) corresponds to the dynamics in the presence of three vortices: it starts after the central vortex is well formed, and finishes before the protrusions of the merged droplet (already largely depleted) accelerate towards the center of the droplet. The second time interval (bottom panels, 150 to $300 \mathrm{ps}$ ) addresses a later time interval with intricate vortex dynamics after the roton burst. The incompressible spectrum remains quite constant over this time period, but the compressible spectrum changes noticeably over the interval, as the roton peak becomes less prominent as times passes.

Finally, Figs. 10 and 11 show the power-law analysis for the dipole configuration. The first time interval (top panels in the figures, 30 to $90 \mathrm{ps}$ ) corresponds to the dynamics where the dark soliton develops, before the droplets merge.

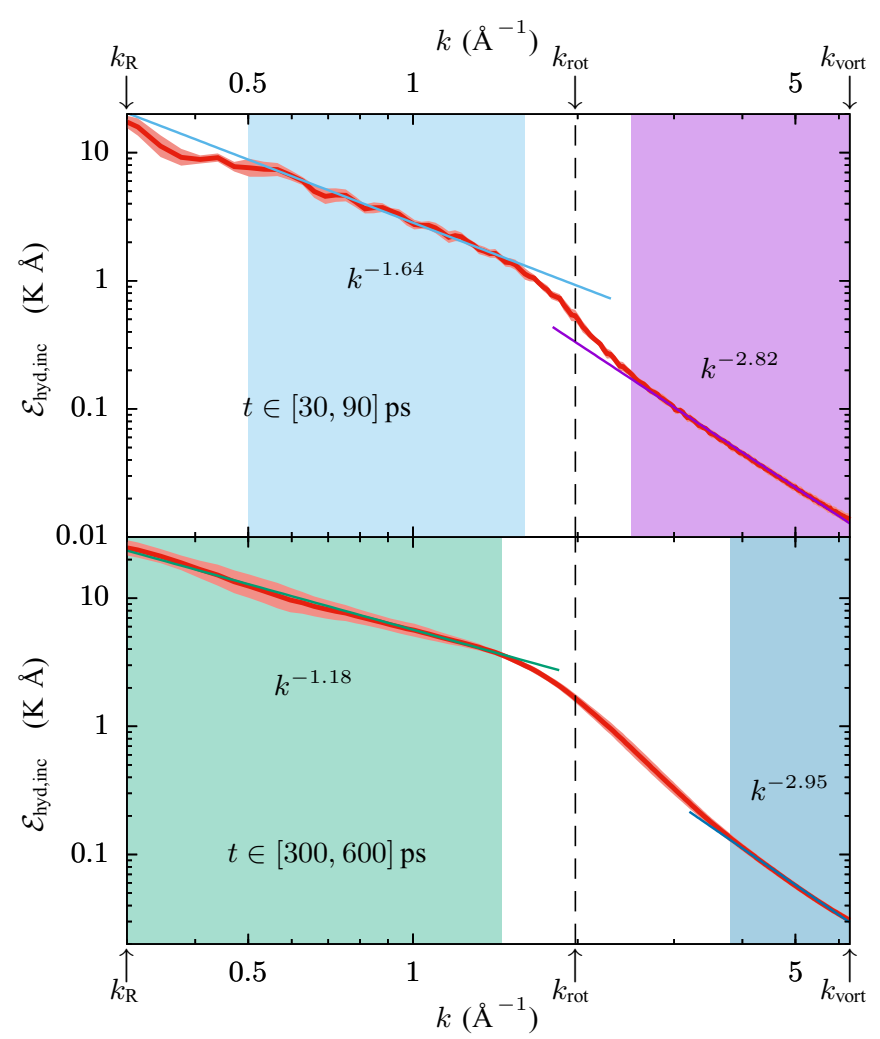

FIG. 10. Power-law analysis of time averages of the incompressible energy spectrum for the dipole configuration. Each of the two panels display, in log-log scales, the time averaged spectrum $\mathcal{E}(k)$ (red line) and the range within a standard deviation (light red) for every $k$, as well as the power laws determined by weighted fits over the shadowed $k$ intervals and indicated time intervals. (Top) $k^{-1.64}$ and $k^{-2.82}$ for $t \in[30,90]$ ps. (Bottom) $k^{-1.18}$ and $k^{-2.95}$ for $t \in[300,600]$ ps.

In the compressible spectrum we have found a progressive widening and strengthening of the roton peak and the possibility of fitting in all cases power laws with exponents close to -3 for large $k$ values. Let us recall that the multimedia materials accompanying this work [18] allows one to dynamically follow the appearance of the compressible and incompressible spectra as a function of time.

We observe that values close to $\alpha=-5 / 3$ and -3 appear in the incompressible spectra of Figs. 8 and 10, which are usually associated, respectively, to the appearance of classical (Kolmogorov) and superfluid turbulence in the bulk [66,70], and to the presence of vortices [66,67]. At variance with superfluid and viscous liquid samples, where the range of available $k$ values extends over decades, in a He droplet or a confined BEC it is rather limited [71], encompassing $k$ values from $\pi / L$, where $L$ is of the order of the radial dimension of the sample, to $\pi / \Delta$, where $\Delta$ is the step used in the space discretization (in our case, 25 and $0.4 \AA$, respectively). As shown in Figs. 8-11, this $k$ range is of the order of a decade (see also Ref. [71],) which makes it difficult to determine if this is a true power-law behavior or just a cross-over.

Yet, in view of the consistency of these scalings, obtained by averaging the power spectrum over fairly large time intervals and not for a single time shot [71], we are prone to 


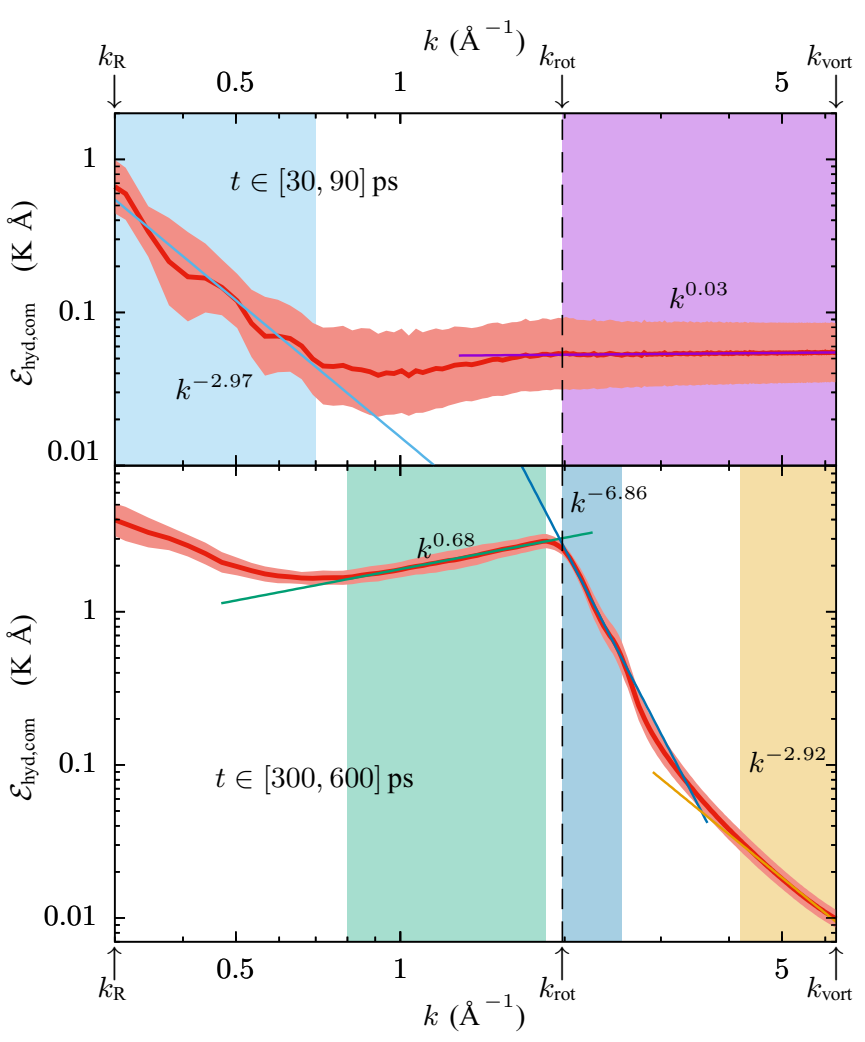

FIG. 11. Power-law analysis of time averages of the compressible energy spectrum for the dipole configuration. Each of the two panels display, in log-log scales, the time averaged spectrum $\mathcal{E}(k)$ (red line) and the range within a standard deviation (light red) for every $k$, as well as the power laws determined by weighted fits over the shadowed $k$ intervals and indicated time intervals. (Top) $k^{-2.97}$ and $k^{0.03}$ for $t \in[30,90]$ ps. (Bottom) $k^{0.68}, k^{-6.86}$, and $k^{-2.92}$ for $t \in[300,600] \mathrm{ps}$.

associate them with the Kolmogorov scenario and with the presence of a vortex core. We want to stress, however, that in general we cannot draw a common and solid explanation for all the observed values of $\alpha$; a deeper understanding of quantum turbulence in the realistic model of ${ }^{4} \mathrm{He}$ that we are using here would imply studying vortex tangles evolution in a much larger drop in order to explore, through the main mechanisms highlighted here (i.e., roton and sound wave emission, and vortex reconnections), the different scales of energy flows, and this is beyond the scope of this paper and current computational capabilities.

\section{SUMMARY}

In this work, we have simulated the coalescence of superfluid helium droplets hosting vortex lines in different relative orientations, finding a rich phenomenology, as, e.g., the appearance of vortex rings slipping along vortex lines and the appearance of dark solitons from the annihilation of vortexantivortex pairs and their decay into vortex rings.

The merging process generates vorticity which adds up to the pre-existing one. In the present study, it builds up in about $100 \mathrm{ps}$ (the time to produce the merged droplet) and it is as large as that originally in the droplets. The generated vorticity is fairly the same irrespective of the relative orientation of the vortex lines in the merging droplets, indicating that its origin has more to do with the droplet dynamics than with the relative orientation of the vortices. This vorticity appears as a genuine surface effect, being especially apparent in the vortex-free case where no vorticity is found in the bulk of the merging droplets during the first $100 \mathrm{ps}$.

We cannot ascertain from our time-limited simulations whether (and how) vorticity fades away. Part of this vorticity disappears by atom evaporation; however, one should have in mind that in spite of being highly excited states of the system, the simultaneous constraints of energy and angular momentum conservation would prevent the decay of vortex lines in a helium droplet [72]. It might thus appear that vortex lines in helium droplets are rather stable. As a matter of fact, they have been detected in large droplets on the millisecond timescale $[25,26]$.

With the experimental realization of stable self-bound ultradilute "quantum droplets" made of bosonic atoms of a binary mixture [73], which have been found to sustain quantum vortices [74], it has been possible to study the collision of self-bound BECs $[13,35]$. Thus it should also be possible to study the merging of quantum droplets and the influence of surface effects in the transfer of angular momentum from one quantum droplet to another, as well as a deeper analysis of the role of the surface protrusions as potential nucleation sites of vortex rings, thus connecting the ultradilute quantum droplet BECs and the superfluid ${ }^{4} \mathrm{He}$ droplets scenarios.

\section{ACKNOWLEDGMENTS}

This work has been performed under Grant No. PID2020-114626GB-I00 from the MICIN/AEI/10.13039/ 501100011033. J.M.E. acknowledges financial support from the Engineering and Physical Sciences Research Council, UK (Grant No. EP/P034616/1); the Spanish Research Agency (AEI) through the Severo Ochoa Centres of Excellence programme (Grant SEV-2017-0706); and the European Union MaX Centre of Excellence (EU-H2020 Grant No. 824143). This work was partly performed under Project HPCEUROPA3 (INFRAIA-2016-1-730897), with the support of the EC Research Innovation Action under the H2020 Programme; in particular, J.M.E. thanks Departament FQA of Universitat de Barcelona for hospitality and acknowledges the computer resources and technical support provided by BSC-CNS.
[1] J. Eggers, J. R. Lister, and H. A. Stone, J. Fluid Mech. 401, 293 (1999).

[2] L. Duchemin, J. Eggers, and C. Josserand, J. Fluid Mech. 487, 167 (2003).
[3] M. Wu, T. Cubaud, and C.-M. Ho, Phys. Fluids 16, L51 (2004).

[4] C. N. Baroud, F. Gallaire, and R. Dangla, Lab. Chip 10, 2032 (2010). 
[5] H. Gu, M. H. G. Duits, and F. Mugele, Int. J. Mol. Sci. 12, 2572 (2011).

[6] J. Qian and C. K. Law, J. Fluid Mech. 331, 59 (1997).

[7] A. Menchaca-Rocha, A. Martínez-Dávalos, R. Núñez, S. Popinet, and S. Zaleski, Phys. Rev. E 63, 046309 (2001).

[8] Y. Pan and K. Sugab, Phys. Fluids 17, 082105 (2005).

[9] K.-L. Pan, P.-C. Chou, and Y.-J. Tseng, Phys. Rev. E 80, 036301 (2009).

[10] K.-L. Huang, K.-L. Pan, and C. Josserand, Phys. Rev. Lett. 123, 234502 (2019).

[11] C. L. Vicente, C. Kim, H. J. Maris, and G. M. Seidel, J. Low Temp. Phys. 121, 627 (2000).

[12] J. M. Escartín, F. Ancilotto, M. Barranco, and M. Pi, Phys. Rev. B 99, 140505(R) (2019).

[13] G. Ferioli, G. Semeghini, L. Masi, G. Giusti, G. Modugno, M. Inguscio, A. Gallemí, A. Recati, and M. Fattori, Phys. Rev. Lett. 122, 090401 (2019).

[14] W. F. Vinen and J. J. Niemela, J. Low Temp. Phys. 128, 167 (2002).

[15] Progress in Low Temperature Physics, edited by M. Tsubota and W. P. Halperin (Elsevier, Amsterdam and London, 2009), Vol. XVI.

[16] C. F. Barenghi, L. Skrbek, and K. P. Sreenivasan, Proc. Natl. Acad. Sci. USA 111, 4647 (2014).

[17] M. Tsubota, K. Fujimoto, and S. Yui, J. Low Temp. Phys. 188, 119 (2017).

[18] See Supplemental Material at http://link.aps.org/supplemental/ 10.1103/PhysRevB.105.024511 for some results corresponding to vortices oriented collinearly and anticollinearly, as well as for the corkscrew configuration. There, one can also find multimedia material displaying the droplet merging for all the initial configurations addressed in this work, namely vortex dimer, dipole, perpendicular, collinear, anticollinear, and corkscrew configurations. For each of them, the multimedia material shows - in a combined way - the time evolution of the droplet densities (2D and 3D), the pseudovorticity $\omega_{\mathrm{ps}}(\mathbf{r}, t)=$ $\nabla \times \mathbf{j}(\mathbf{r}, t)$ visualized by the $\left\|\omega_{\mathrm{ps}}(\mathbf{r}, t)\right\|=10^{-3} \mathrm{ps}^{-1}$ isosurfaces, and the compressible and incompressible energy spectra as a function of the wave number $k$.

[19] F. Ancilotto, M. Barranco, F. Coppens, J. Eloranta, N. Halberstadt, A. Hernando, D. Mateo, and M. Pi, Int. Rev. Phys. Chem. 36, 621 (2017).

[20] L. Pitaevskii and S. Stringari, Bose-Einstein Condensation and Superfluidity, International Series of Monographs on Physics Vol. 164 (Oxford University Press, Oxford, 2016).

[21] B. Sun and M. S. Pindzola, J. Phys. B: At. Mol. Opt. Phys. 41, 155302 (2008).

[22] D. Mateo, A. Leal, A. Hernando, M. Barranco, M. Pi, F. Cargnoni, M. Mella, X. Zhang, and M. Drabbels, J. Chem. Phys. 140, 131101 (2014).

[23] A. Leal, D. Mateo, A. Hernando, M. Pi, M. Barranco, A. Ponti, F. Cargnoni, and M. Drabbels, Phys. Rev. B 90, 224518 (2014).

[24] F. Coppens, F. Ancilotto, M. Barranco, N. Halberstadt, and M. Pi, Phys. Chem. Chem. Phys. 19, 24805 (2017).

[25] L. F. Gomez, K. R. Ferguson, J. P. Cryan, C. Bacellar, R. M. P. Tanyag, C. Jones, S. Schorb, D. Anielski, A. Belkacem, C. Bernando, R. Boll, J. Bozek, S. Carron, G. Chen, T. Delmas, L. Englert, S. W. Epp, B. Erk. L. Foucar, R. Hartmann, A.
Hexemer, M. Huth, J. Kwok, S. R. Leone, J. H. S. Ma, F. R. N. C. Maia, E. Malmerberg, S. Marchesini, D. M. Neumark, B. Poon, J. Prell, D. Rolles, B. Rudek, A. Rudenko, M. Seifrid, K. R. Siefermann, F. P. Sturm, M. Swiggers, J. Ullrich, F. Weise, P. Zwart, C. Bostedt, O. Gessner, and A. F. Vilesov, Science 345, 906 (2014).

[26] S. M. O. O’Connell, R. M. P. Tanyag, D. Verma, C. Bernando, W. Pang, C. Bacellar, C. A. Saladrigas, J. Mahl, B. W. Toulson, Y. Kumagai, P. Walter, F. Ancilotto, M. Barranco, M. Pi, C. Bostedt, O. Gessner, and A. F. Vilesov, Phys. Rev. Lett. 124, 215301 (2020).

[27] M. Pi, F. Ancilotto, F. Coppens, N. Halberstadt, A. Hernando, A. Leal, D. Mateo, R. Mayol, and M. Barranco, 4He-DFT BCN-TLS: A Computer Package for Simulating Structural Properties and Dynamics of Doped Liquid Helium-4 Systems. https://github.com/bentls2016/

[28] M. Barranco, F. Coppens, N. Halberstadt, A. Hernando, A. Leal, D. Mateo, R. Mayol, and M. Pi, Zero temperature DFT and TDDFT for ${ }^{4} \mathrm{He}$ : A short guide for practitioners (2017), https: //github.com/bcntls2016/DFT-Guide/blob/master/dft-guide.pdf

[29] F. Ancilotto, M. Barranco, F. Caupin, R. Mayol, and M. Pi, Phys. Rev. B 72, 214522 (2005).

[30] D. E. Galli, L. Reatto, and M. Rossi, Phys. Rev. B 89, 224516 (2014).

[31] D. Mateo, J. Eloranta, and G. A. Williams, J. Chem. Phys. 142, 064510 (2015).

[32] I. Amelio, D. E. Galli, and L. Reatto, Phys. Rev. Lett. 121, 015302 (2018).

[33] F. Dalfovo, R. Mayol, M. Pi, and M. Barranco, Phys. Rev. Lett. 85, 1028 (2000).

[34] M. Barranco, R. Guardiola, S. Hernández, R. Mayol, J. Navarro, and M. Pi, J. Low Temp. Phys. 142, 1 (2006).

[35] V. Cikojević, L. Vranješ Markić, M. Pi, M. Barranco, F. Ancilotto, and J. Boronat, Phys. Rev. Research 3, 043139 (2021).

[36] F. Ancilotto, M. Barranco, and M. Pi, Phys. Rev. B 97, 184515 (2018).

[37] M. Pi, J. M. Escartín, and F. Ancilotto, and M. Barranco, Phys. Rev. B 104, 094509 (2021)

[38] K. Kono, S. Kobayashi, and W. Sasaki, J. Phys. Soc. Jpn. 50, 721 (1981).

[39] M. J. McKenna, R. J. Stanley, E. DiMasi, and J. D. Maynard, Phys. B: Condens. Matter 165-166, 603 (1990).

[40] V. A. Hopkins, J. Keat, G. D. Meegan, T. Zhang, and J. D. Maynard, Phys. Rev. Lett. 76, 1102 (1996).

[41] A. A. Levchenko, G. V. Kolmakov, L. P. Mezhov-Deglin, and V. B. Shikin, JETP Lett. 65, 572 (1997).

[42] F. Ancilotto, D. Levy, J. Pimentel, and J. Eloranta, Phys. Rev. Lett. 120, 035302 (2018).

[43] X. Buelna, D. Gonzalez, A. Freund, and J. Eloranta, Phys. Rev. B 99, 144518 (2019).

[44] S. Burger, K. Bongs, S. Dettmer, W. Ertmer, K. Sengstock, A. Sanpera, G. V. Shlyapnikov, and M. Lewenstein, Phys. Rev. Lett. 83, 5198 (1999).

[45] J. Denschlag, J. E. Simsarian, D. L. Feder, C. W. Clark, L. A. Collins, J. Cubizolles, L. Deng, E. W. Hagley, K. Helmerson, W. P. Reinhardt, S. L. Rolston, B. I. Schneider, and W. D. Phillips, Science 287, 97 (2000). 
[46] B. P. Anderson, P. C. Haljan, C. A. Regal, D. L. Feder, L. A. Collins, C. W. Clark, and E. A. Cornell, Phys. Rev. Lett. 86, 2926 (2001).

[47] C. Becker, S. Stellmer, P. Soltan-Panahi, S. Dorscher, M. Baumert, E.-M. Richter, J. Kronjger, K. Bongs, and K. Sengstock, Nat. Phys. 4, 496 (2008).

[48] D. R. Scherer, C. N. Weiler, T. W. Neely, and B. P. Anderson, Phys. Rev. Lett. 98, 110402 (2007).

[49] A. E. Muryshev, H. B. van Linden van den Heuvell, and G. V. Shlyapnikov, Phys. Rev. A 60, R2665 (1999).

[50] D. L. Feder, M. S. Pindzola, L. A. Collins, B. I. Schneider, and C. W. Clark, Phys. Rev. A 62, 053606 (2000).

[51] M. J. H. Ku, B. Mukherjee, T. Yefsah, and M. W. Zwierlein, Phys. Rev. Lett. 116, 045304 (2016).

[52] S. Donadello, S. Serafini, M. Tylutki, L. P. Pitaevskii, F. Dalfovo, G. Lamporesi, and G. Ferrari, Phys. Rev. Lett. 113, 065302 (2014).

[53] T. Kanai, W. Guo, M. Tsubota, and D. Jin, Phys. Rev. Lett. 124, 105302 (2020).

[54] R. J. Donnelly, Quantized vortices in helium II, Cambridge Studies in Low Temperature Physics Vol. 3 (Cambridge University Press, Cambridge, UK 1991).

[55] A. Villois, G. Krstulovic, D. Proment, and H. Salman, J. Phys. A: Math. Theor. 49, 415502 (2016).

[56] C. Falcón, E. Falcon, U. Bortolozzo, and S. Fauve, Europhys. Lett. 86, 14002 (2009).

[57] S. Nazarenko, Contemp. Phys. 56, 359 (2015).

[58] C. F. Barenghi, R. J. Donnelly, and W. F. Vinen, Quantized Vortex Dynamics and Superfluid Turbulence (Springer, Berlin, 2001).

[59] A. Villois and H. Salman, Phys. Rev. B 97, 094507 (2018).
[60] N. G. Berloff and P. H. Roberts, J. Phys. A 34, 81 (2001).

[61] T. Frisch, Y. Pomeau, and S. Rica, Phys. Rev. Lett. 69, 1644 (1992).

[62] N. Navon, A. L. Gaunt, R. P. Smith, and Z. Hadzibabic, Nature (London) 539, 72 (2016).

[63] N. G. Berloff and B. V. Svistunov, Phys. Rev. A 66, 013603 (2002).

[64] M. Kobayashi and M. Tsubota, Phys. Rev. Lett. 94, 065302 (2005).

[65] D. Proment, S. Nazarenko, and M. Onorato, Phys. Rev. A 80, 051603(R) (2009).

[66] C. Nore, M. Abid, and M. E. Brachet, Phys. Fluids 9, 2644 (1997).

[67] A. S. Bradley and B. P. Anderson, Phys. Rev. X 2, 041001 (2012).

[68] M. T. Reeves, B. P. Anderson, and A. S. Bradley, Phys. Rev. A 86, 053621 (2012).

[69] R. McKeown, R. Ostilla-Mónico, A. Pumir, M. P. Brenner, and S. M. Rubinstein, Phys. Rev. Fluids 3, 100509 (2018).

[70] J. Salort, C. Baudet, B. Castaing, B. Chabaud, F. Daviaud, T. Didelot, P. Diribarne, B. Dubrulle, Y. Gagne, F. Gauthier, A. Guirard, B. Hébral, B. Rousset, P. Thibault, and P.-E. Roche, Phys. Fluids 22, 125102 (2010).

[71] A. Cidrim, A. C. White, A. J. Allen, V. S. Bagnato, and C. F. Barenghi, Phys. Rev. A 96, 023617 (2017).

[72] K. K. Lehmann and R. Schmied, Phys. Rev. B 68, 224520 (2003).

[73] C. R. Cabrera, L. Tanzi, J. Sanz, B. Naylor, P. Thomas, P. Cheiney, and L. Tarruell, Science 359, 301 (2018).

[74] F. Ancilotto, M. Barranco, M. Guilleumas, and M. Pi, Phys. Rev. A 98, 053623 (2018). 